\title{
Nested long period grating interferometers
}

\author{
Richard P. Murphy, Stephen W. James and Ralph P. Tatam \\ Engineering Photonics Group, School of Engineering, Cranfield University \\ Cranfield, Bedford, MK43 0AL, UK
}

\begin{abstract}
The concept of nested fibre optic long period grating (LPG) based interferometers is introduced. A number of in-series, identical LPGs may be used to form a set of nested, multiplexed Mach-Zehnder interferometers that may demodulated and demultiplexed by virtue of a Fourier analysis of the optical spectrum. The concept is demonstrated by the use of three LPGs to form a nested set of interferometers.
\end{abstract}

Keywords: optical fibre long period grating, nested interferometer

\section{INTRODUCTION}

The core-cladding mode coupling facilitated by a fibre optic long period grating (LPG) offers the prospect for development of sensors whose principle of operation is based on the interaction of the cladding mode with the surrounding environment. This offers particular advantages for concepts that exploit the evanescent field of waveguide modes, for example refractometry ${ }^{1}$ and systems in which functional coatings are deposited on the fibre ${ }^{2}$, as it eliminates the requirement to access the evanescent field of the core mode through removal of the cladding by polishing, etching or micromachining.

An LPG is a periodic modulation of the propagation constants of the modes of an optical fibre, formed by a number of means, particularly UV-induced modulation of the refractive index of the core of the fibre. The period of the modulation is typically in the range $100 \mu \mathrm{m}$ to $1000 \mu \mathrm{m}$, and facilitates coupling of energy between the propagating mode of the core of the fibre and a discreet set of forward-propagating cladding modes ${ }^{3}$. Coupling takes place at wavelengths, $\lambda_{v}$ determined by the phase matching condition, being dependent on the product of the modulation period and the difference between the effective indices of the core and cladding modes ${ }^{3}$,

$$
\lambda_{i}=\left(n_{\text {eff }}(\lambda)-n_{c l}^{i}(\lambda)\right) \Lambda
$$

where $n_{\text {eff }}(\lambda)$ is the effective refractive index of the propagating core mode at wavelength $\lambda, n_{c l}^{i}(\lambda)$ is the refractive index of the $\mathrm{i}^{\text {th }}$ cladding mode and $\Lambda$ is the period of the LPG. The high attenuation of the cladding modes results in the presence of a set of attenuation bands at central wavelengths defined by equation (1).

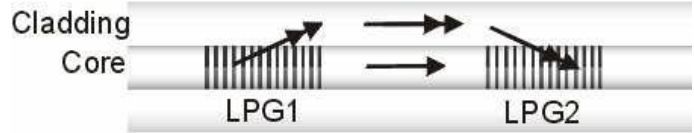

(a)

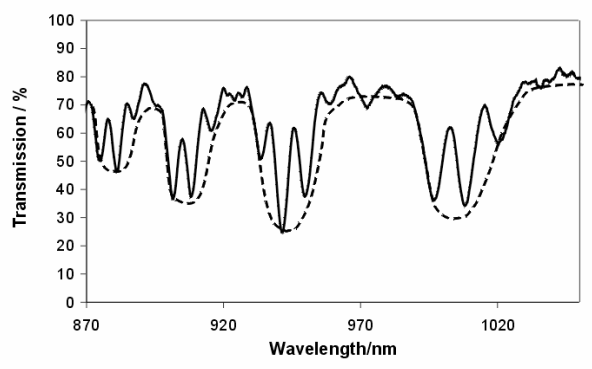

(b)

Figure 1. (a) Operation of cascaded LPGs as an MZI (b) channeled spectra in the attenuation bands

Third European Workshop on Optical Fibre Sensors, Antonello Cutolo, Brian Culshaw, José Miguel López-Higuera, Eds. Proceedings of SPIE Vol. 6619, 66193G, (2007) · 0277-786X/07/\$18 $\cdot$ doi: 10.1117/12.738589 
The fabrication of in-series or cascaded LPGs forms an in-fibre Mach-Zehnder interferometer (MZI), as illustrated in figure 1. This introduces a sinusoidal channeled spectrum into the attenuation bands. The frequency of the sinusoid is determined by the physical separation of the LPGs. Changes in the phase of the sinusoid may be introduced by a differential change between the effective indices of the core and cladding modes, induced by external perturbations such as changes in the surrounding refractive index. This has been exploited recently as a means to multiplex and demodulate a serial array of cascaded LPGs ${ }^{4}$.

In this paper a means for forming a set of nested, multiplexed, Mach-Zehnder interferometers by the use of a serial array of identical LPGs is proposed. The interferometers may be demodulated and demultiplexed by virtue of a Fourier analysis of the optical spectrum. The system provides a number of uniquely identifiable interferometric sensors operating with different spatial resolutions, or a number of interferometers that may be configured to monitor different measurands, for example by depositing different functional coatings on the fibre length separating adjacent LPGs. The concept is demonstrated by the use of three LPGs to form a set of nested interferometers.

\section{PRINCIPLE}

When a serial array of LPGs with identical characteristics is formed in a length of optical fibre that is stripped of its buffer jacket, a set of nested interferometers is formed, with each LPG interacting with all other LPGs in the array. Appropriate design of the physical separation of the LPGs within the array allows each interferometer to generate a channeled spectrum with a unique frequency, the phase of which provides information on the environment surrounding the relevant fibre lengths.

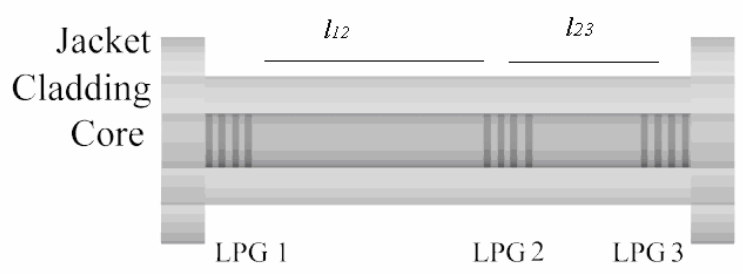

Figure 2. Nested MZI interferometers formed between 3 in-series LPGs

To demonstrate the principle, a serial array of three identical LPGs is considered, as illustrated in figure 2. The adjacent LPGs are separated by distances $l_{12}$ and $l_{23}$. Figure 3 illustrates the six combinations of beam paths from which the interferometers are formed. Interferometers are formed between the adjacent LPGs, between the extreme LPG elements in the array, and a compound interferometer that involves all three LPGs is formed. An analysis of the system reveals that the transmission spectrum contains four frequencies, given by

$$
\begin{gathered}
f_{12}=k\left(n_{e f f}^{c o}-n_{e f f}^{c l}\right)_{12} l_{12} \\
f_{23}=k\left(n_{e f f}^{c o}-n_{e f f}^{c l}\right)_{23} l_{23} \\
f_{\Sigma}=k\left(n_{e f f}^{c o}-n_{e f f}^{c l}\right)_{12} l_{12}+\left(n_{e f f}^{c o}-n_{e f f}^{c l}\right)_{23} l_{23} \\
f_{\Delta}=k\left(n_{e f f}^{c o}-n_{e f f}^{c l}\right)_{12} l_{12}-\left(n_{e f f}^{c o}-n_{e f f}^{c l}\right)_{23} l_{23}
\end{gathered}
$$

where $k$ is the wavevector, $2 \pi / \lambda$, and the subscripts 12 and 23 refer to the lengths of fibre separating LPGs 1 and 2, and LPGs 2 and 3, respectively. Thus, with suitable choice of $l_{12}$ and $l_{23}$, it is possible to uniquely identify and, by determining the changes in phase at these frequencies, to interrogate the interferometers formed between adjacent LPGs, and to also measure directly the sum and differences of the responses of the two interferometers. 


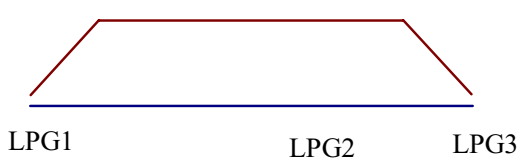

(a)
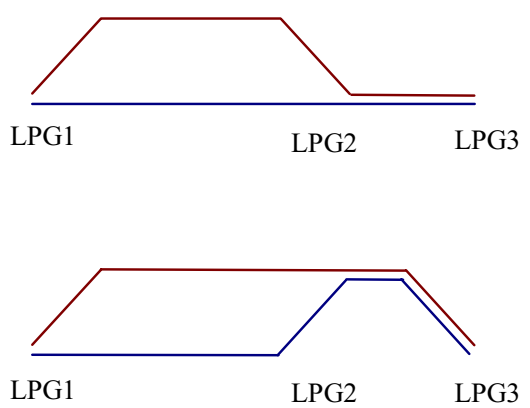

(c)

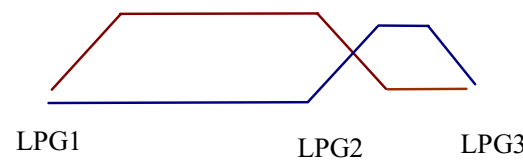

(b)
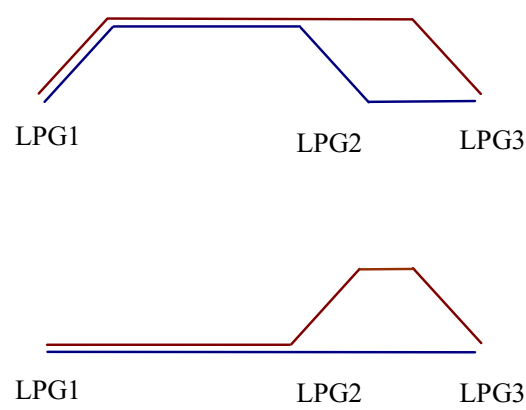

(d)

Figure 3. (a) Beam path for the interferometer generating $f_{\Sigma}$. (b) Beam path for the interferometer generating frequency $f_{\Delta}$. (c)Beam paths for the interferometer generating frequency $f_{12}$ (d) Beam paths for the interferometer generating frequency $f_{23}$.

\section{EXPERIMENT AND RESULTS}

To verify the operation of the proposed nested interferometers, the buffer jacket was removed from a $180 \mathrm{~mm}$ length of a hydrogen loaded single mode optical fibre with a cutoff wavelength of $650 \mathrm{~nm}$. Three LPGs, each of length $30 \mathrm{~mm}$, were fabricated via UV irradiation though an amplitude mask of period of $400 \mu \mathrm{m}$. The transmission spectrum was monitored by coupling the output from a tungsten-halogen lamp into the fibre, and coupling the output of the fibre to a CCD spectrometer (Ocean Optics HR4000). The length of fibre separating the LPGs were $l_{12}=30 \mathrm{~mm}$, and $l_{23}=90 \mathrm{~mm}$. The transmission spectrum is shown in figure 4 (a) and the corresponding Fourier Transform is shown in figure 4 (b), with the frequencies of interest labeled.

The sections of fibre separating the LPGs were immersed in wells containing a Cargille oil of refractive index 1.46. The temperature of the oil in the wells was controlled using a thermo-electric cooler. Changing the temperature of the wells induced a change in the phase of the interferometers via two mechanisms, firstly through the differential thermal response of the effective indices of the core and cladding modes, and secondly through the thermo-optically induced change in the refractive index of the oil, which resulted in a concomitant change in the effective index of the cladding mode.

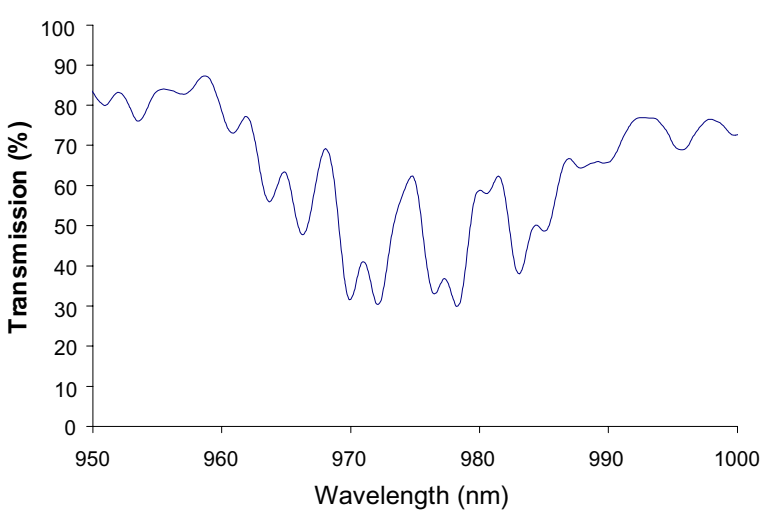

(a)

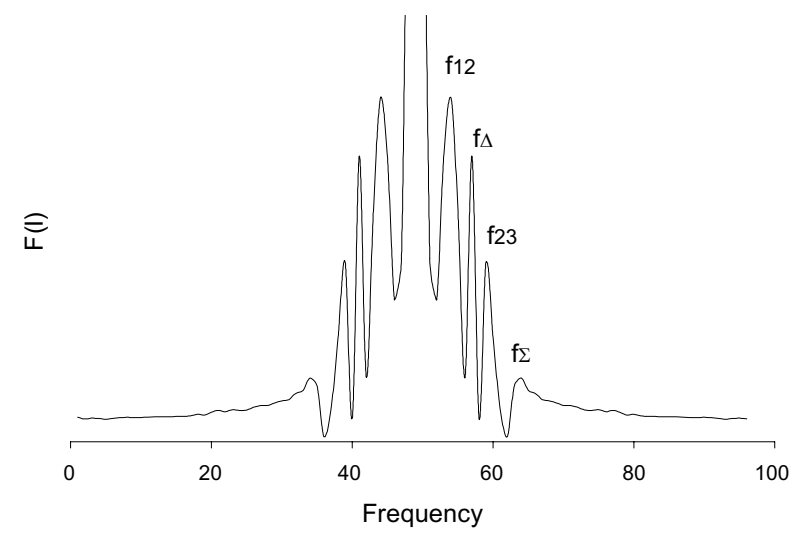

(b)

Figure 4. (a) Transmission spectrum of the serial array of three LPGS, (b) Fourier transform of the spectrum. The frequencies $f_{12}, f_{23}, f_{\Sigma}$ and $f_{\Delta}$, are those identified in equations $(5)-(8)$. 
The frequencies of interest were isolated in the Fourier Transform of the transmission spectrum and, after translation to the origin, the arctangent of the ratio of the real to imaginary components was used to determine the phase ${ }^{5}$. The phase values obtained in this way were wrapped modulo $2 \pi$, resulting in discontinuities in the phase, which were then unwrapped.

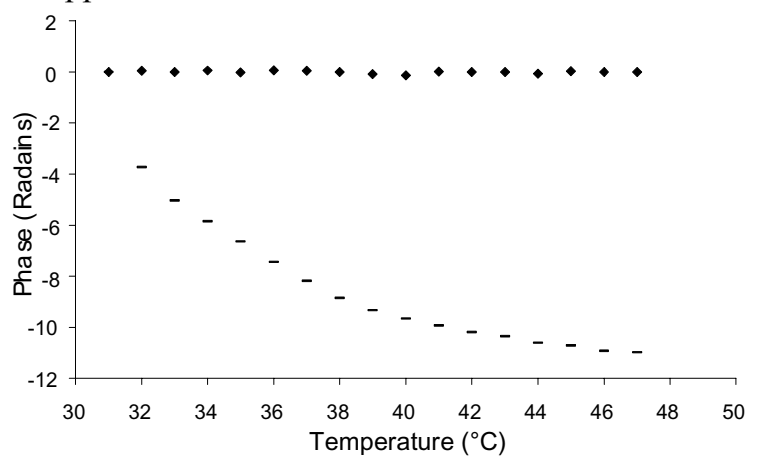

(a)

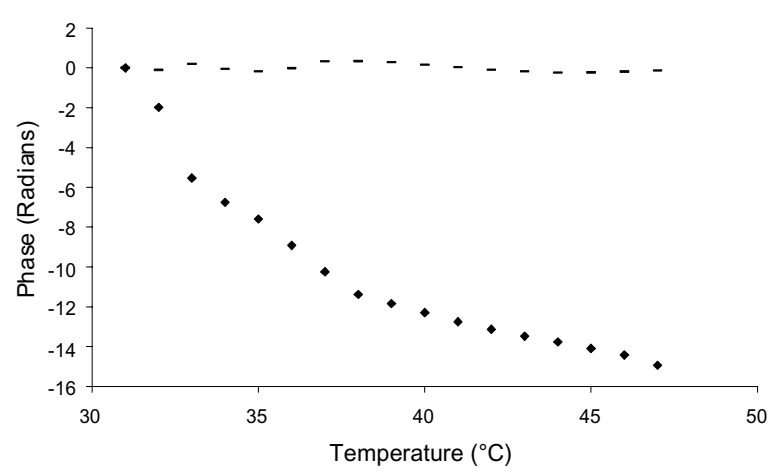

(b)

Figure 5. (a) Change of phase of the fringe patterns in response to increasing the temperature of Cargille oil surrounding a section of the fibre separating adjacent LPGs. (a) LPG 1 and LPG 2, and (b) LPG 2 and LPG3. -, phase of fringes corresponding to the MZI formed between LPG 1 and LPG 2; , phase of fringes corresponding to the MZI formed between LPG 2 and LPG 3.

The results shown in figure 5(a) were obtained when the temperature of the oil bath surrounding length of fibre separating LPGs 1 and 2 was varied, while those in 5 (b) were obtained when the temperature of the oil bath surrounding length of fibre separating LPGs 2 and 3 was varied. The phases of the interferometers defined by LPG 1 and LPG2, and by LPG 2 and LPG 3 can be measured independently using this technique. In both figures, the drift in the phase of the fringes corresponding to the interferometer section held at constant temperature equates to a variation of the temperature of the oil of $\pm 0.1^{\circ} \mathrm{C}$, which lies within the temperature stability achieved by the thermoelectric controller.

\section{SUMMARY}

In summary, a serial array of 3 LPGs has been used to demonstrate the concept of nested LPG based Mach-Zehnder interferometers. Fourier analysis of the transmission spectrum allows the identification of the interferometers formed by the in-series LPGs, and facilitates the determination of measurand induced phase shifts. The number of interferometers that may be interrogated in this way is limited by the resolution of the method used to measure the transmission spectrum, and by the losses of the cladding modes.

The Authors would like to acknowledge the support of the Engineering and Physical Science Research Council UK for their support.

\section{REFERENCES}

1. S W James and R P Tatam, "Optical Fibre Long Period Grating Sensors: Characteristics and Application", Meas.Sci.Technol. 14 , R49 (2003)

2. H J Patrick, A D Kersey and F Bucholtz "Analysis of the response of long period fibre gratings to external index of refraction" J. Lightwave Technol.16 1606 (1998)

3. S W James, N D Rees, G J Ashwell and R P Tatam "Optical fibre long period gratings with Langmuir Blodgett thin film overlays" Opt. Lett. 9686 (2002)

4. V Bhatia and A M Vengsarkar "Optical fibre long-period grating sensors" Opt. Lett. 21692 (1996)

5. R P Murphy, S W James and R P Tatam "Multiplexing of fibre optic long period grating based interferometric sensors", J. Lightwave Technol. in press (1007)

6. M. Takeda, H. Ina, S. Kobayashi, "Fourier-transform method of fringe-pattern analysis for computer-based topography and interferometry”, J. Opt. Soc. Am, 72, 156 (1982). 